\title{
Experiencia del Centro de Simulación de la Facultad de Medicina de la Universidad de Navarra
}

\author{
Javier. Pueyo ${ }^{1, *}$, Cristina Honorato ${ }^{2}$, Nekane Uruñuela ${ }^{3}$, David Valencia ${ }^{4}$, Rubén Orio ${ }^{4}$, Juan \\ Carlos Díez ${ }^{4}$, Nieves Díez ${ }^{5}$, Juan Alcalde ${ }^{6}$, Secundino Fernández ${ }^{7}$, Nerea Martín ${ }^{8}$ \\ 1 Director del CSM, Facultad de Medicina de la Universidad de Navarra; ORCID id: https://orcid.org/0000- \\ 0002-4369-5610 \\ 2 Subdirectora del CSM y Coordinadora de Postgrado, Facultad de Medicina de la Universidad de Navarra; \\ ORCID id: https://orcid.org/0000-0002-5177-6285 \\ 3 Gestora del Centro de Simulación, Facultad de Medicina de la Universidad de Navarra \\ 4 Técnicos del Centro de Simulación, Facultad de Medicina de la Universidad de Navarra \\ 5 Coordinadora de Grado, Centro de Simulación, Facultad de Medicina de la Universidad de Navarra; \\ ORCID id: https://orcid.org/0000-0002-6564-5091 \\ 6 Coordinador de Innovación, Centro de Simulación, Facultad de Medicina de la Universidad de Navarra; \\ ORCID id: https://orcid.org/0000-0002-6365-3100 \\ 7 Decano de la Facultad de Medicina, Facultad de Medicina de la Universidad de Navarra; ORCID id: \\ https://orcid.org/0000-0002-3858-0034 \\ 8 Coordinadora de Investigación, Facultad de Medicina de la Universidad de Navarra; ORCID id: \\ https://orcid.org/0000-0001-7549-1455 \\ * Autor correspondencia: jpuevi@unav.es; \\ DOI: https://doi.org/10.37536/RIECS.2021.6.2.294
}

\section{Introducción}

En los últimos años, las facultades de Medicina se han visto en la necesidad de ofrecer cambios en su forma de impartir la docencia; los alumnos, los pacientes y la normativa han cambiado. Estas circunstancias obligan a una renovación permanente con el objetivo de innovar y alcanzar la excelencia en la docencia universitaria.

Fruto de esa renovación, la simulación ha adquirido un papel fundamental en la formación en el Grado de Medicina. La simulación ofrece un entorno seguro y controlado en el que reproducir, tantas veces como sea necesario, situaciones en las que el estudiante va construyendo su aprendizaje a partir de un trabajo explicativo, reflexivo, vivencial y colaborativo. Por otro lado, los avances tecnológicos hacen que las herramientas docentes en simulación sean altamente cambiantes, lo que obliga a una actualización permanente del equipo técnico y del equipo docente.

En este monográfico acerca de la simulación en el Grado de Medicina se expondrá la experiencia del Centro de Simulación de la Facultad de Medicina (CSM) de la Universidad de Navarra, que se inauguró en 2011. 
2. Funcionamiento general del Centro de Simulación de la Facultad de Medicina en la Universidad de Navarra

La siguiente figura muestra el organigrama del CSM en la actualidad:

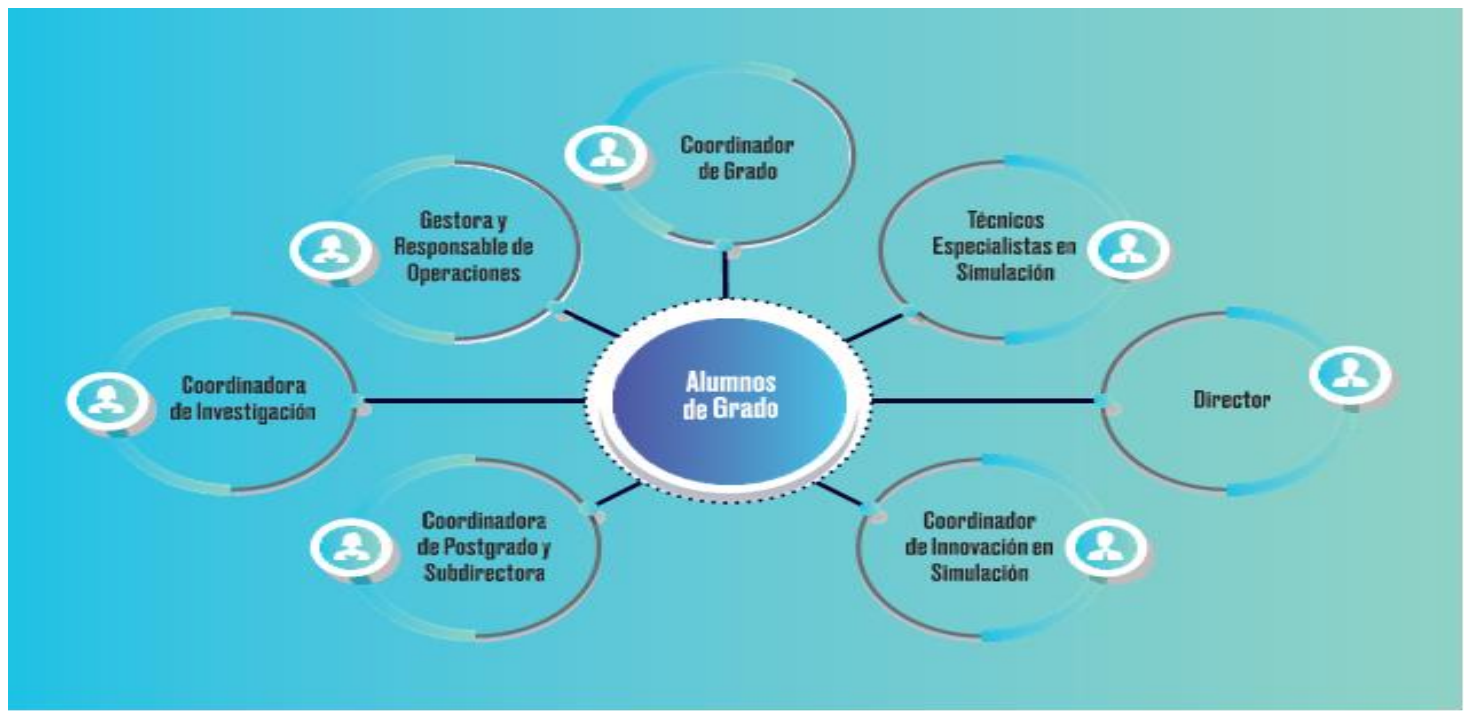

Figura 1

Cada integrante del equipo tiene unas funciones específicas y un perfil profesional muy concreto, así como unas aptitudes que consideramos necesarias. Excepto la gestora y los técnicos, que trabajan a tiempo completo, el resto de miembros trabaja a tiempo parcial. Todos ellos deben estar inmersos en un proceso de formación continuada.

Las alumnas y los alumnos están en el centro de todo. Todo nuestro trabajo gira en torno a ellos.

El director y la subdirectora del centro son los responsables de la toma de decisiones, y todas las propuestas deben ser aprobadas por ellos, aunque las decisiones estratégicas son tomadas de forma colegiada por toda la Junta. Deben ser personas empáticas, con habilidades de comunicación, capacidad de análisis y resolución de problemas, gestión de recursos y de personal, visión de futuro.

La coordinadora de grado es la encargada de diseñar nuevas guías, talleres, escenarios... Este centro de simulación está enfocado, mayoritariamente, a la docencia de grado. La renovación de los planes de estudios obliga a una actualización permanente de la simulación como herramienta docente, por lo que la coordinadora de grado es una figura clave en el funcionamiento de nuestro centro.

El coordinador de innovación en simulación es el encargado de conocer las novedades en publicaciones y evidencias científicas, así como novedades del mercado, nuevos materiales y analizar su idoneidad para las actividades que se llevan a cabo en el CSM. Debe ser una persona proactiva, interesada por las nuevas tecnologías y capacidad de análisis crítico.

La coordinadora de investigación planifica, dirige y coordina las actividades de investigación que se llevan a cabo desde el CSM. Estas están encaminadas actualmente al desarrollo de Trabajos de Fin de Grado y Proyectos de Innovación Docente, y su extensión a tesis doctorales en colaboración con el equipo de la Unidad de Educación Médica. Debe ser una persona con formación en metodología de la investigación, capacidad de trabajo en equipo y buenas habilidades de comunicación.

La gestora es la responsable de planificar, ejecutar y controlar a los equipos. Conoce los objetivos del plan de desarrollo y se asegura de que los equipos trabajen en la dirección adecuada. Debe ser una persona con capacidad de liderazgo, resolutiva, que sepa trabajar en equipo y tenga buenas habilidades de comunicación.

El equipo de técnicos especialistas en simulación proporciona asistencia administrativa y técnica en el centro de simulación. Colaboran estrechamente en tareas docentes y de investigación, así como de gestión del stock y almacén de materiales en el centro. Es muy importante su formación en lo que llamamos las 6 áreas del conocimiento que todo técnico de simulación debe tener. 


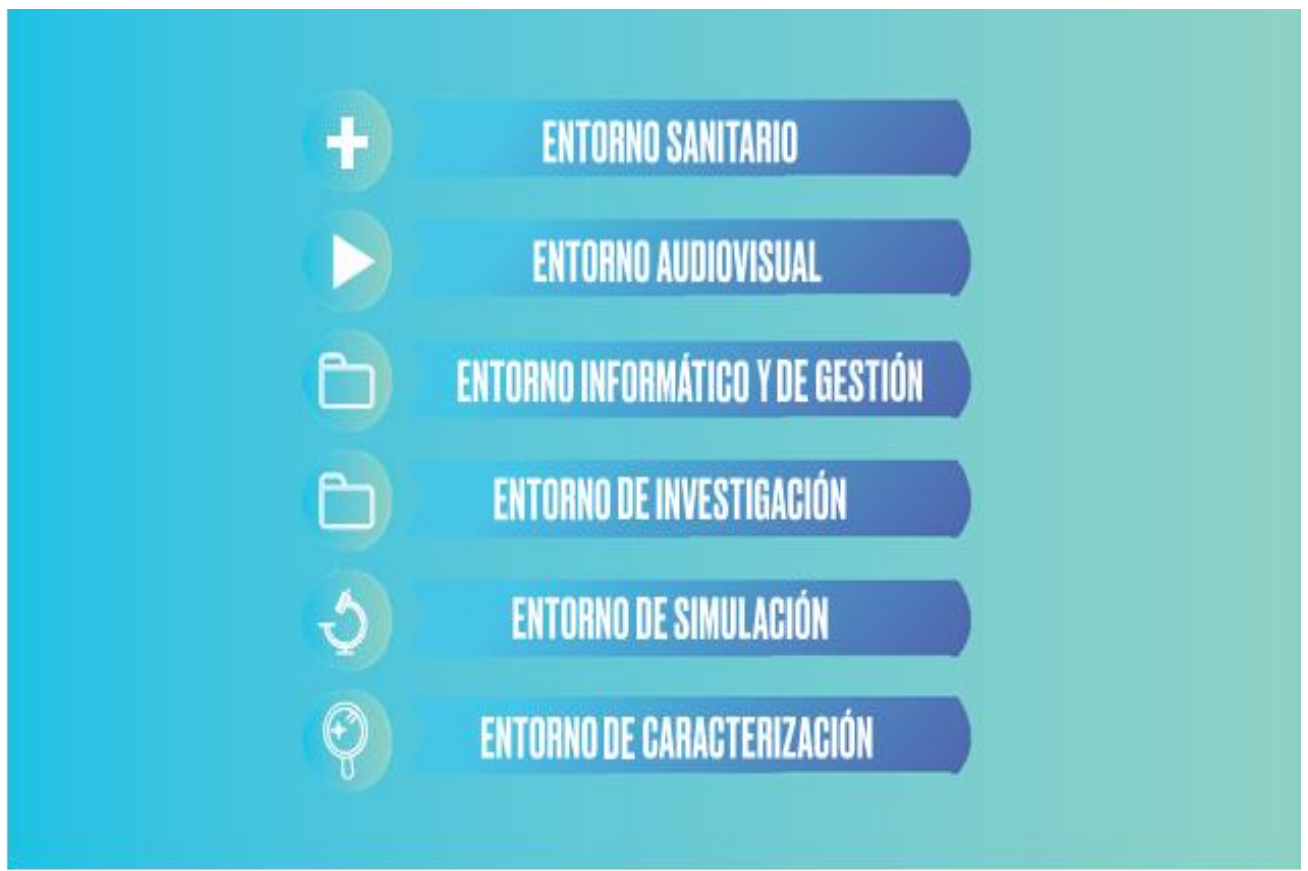

Figura 2

Se requiere del equipo de técnicos en simulación:

- Conocimiento básico de la estructura de la enseñanza universitaria, los principios que la rigen, y los objetivos docentes, así como de las diversas metodologías docentes.

- Conocimiento básico del entorno sanitario y su estructura de funcionamiento.

- Formación básica de anatomía, fisiología, farmacología, terminología médica y material sanitario.

- Capacidad de gestión humana: trabajo en equipo, comunicación, gestión del tiempo, priorización de tareas.

- Capacidad de gestión de material y técnica: inventario, adquisición y mantenimiento de simuladores, software de programación de simuladores, producción de consumibles, preparación y adecuación de aulas para actividades (organización de espacios polivalentes, montaje de simuladores y material fungible).

- Conocimiento de la normativa del centro y de la Facultad, incluyendo protección de datos, control de accesos, cumplimiento de medidas de seguridad, etc.

- Habilidad en la producción y edición avanzada de vídeo en tiempo casi real.

- Capacidad de resolución de problemas con el material de filmación y audio.

- Habilidades en la producción y realización de talleres y escenarios online con desarrollo e interacción remota en streaming.

- Conocimientos básicos generales de los principios y métodos de investigación cualitativa.

- Conocimiento en los principios básicos generales de funcionamiento de la simulación: estructura, valor, diseño de actividades, fundamentos pedagógicos.

- Capacidad de apoyar la realización de escenarios mediante teatralización, puesta en escena, moulage, apoyo técnico al profesorado, sistemas audiovisuales.

- Conocimientos básicos de briefing y debriefing (elementos estructurales pedagógicos de los escenarios de simulación).

- Estructura y principios de la educación interprofesional.

- Formación y trabajo conjunto con pacientes estandarizados.

Todos estos requisitos hacen de los técnicos en simulación un personal con unas características y preparación concretas. En España ahora mismo no existe una formación específica. La mayoría de los técnicos proviene de entornos de electrónica, ingeniería o gestión, y reciben formación a medida que desempañan su trabajo, dependiendo de las necesidades concretas del centro en que trabajen. 
En la línea de aprovechar al máximo los simuladores, los técnicos tienen mucho que aportar en la decisión de adquirir un simulador nuevo ya que, si no va a usarse bien o no se adecúa a las necesidades docentes, es fácil que acaben enterrados en un armario.

\section{La importancia de la metodología y la formación del profesorado}

La consecución de los objetivos docentes depende, en gran medida, de la elección adecuada de la herramienta docente y de la adecuada formación del profesorado.

Nuestro objetivo es que en todas las asignaturas de ámbito clínico se utilice la simulación como herramienta docente. Cuando el profesorado está de acuerdo, se convoca una reunión donde se decide conjuntamente qué metodología emplear y sentar las bases de la colaboración, desde la planificación logística y pedagógica hasta su ejecución material. Entre las distintas herramientas de docencia en simulación ofrecemos: talleres para el aprendizaje y entrenamiento de habilidades, prácticas con modelo humano y escenarios con simulador o paciente estandarizado; que abarquen no sólo aspectos técnicos si no también los no técnicos (liderazgo, coordinación de equipo, comunicación efectiva, manejo de estrés...), permitiendo el entrenamiento individual o en equipo. Es en dicha reunión cuando se establecen los objetivos de aprendizaje, que constituyen la base de nuestro trabajo y que deben estar bien definidos y estructura- dos. Una vez expuestos los objetivos de aprendizaje y las competencias que el alumno debe adquirir, se establece la herramienta que mejor se adecúe a las necesidades del profesor y, muy importante, al tiempo del que se dispone.

Una vez definidos los objetivos y las herramientas, se inicia la fase de trabajo conjunto que culminará con el desarrollo de las actividades propuestas, aunque se mantiene un proceso permanente de evaluación y mejora. Para sistematizar y facilitar ese trabajo se emplea una "hoja de ruta" (Anexo 1) que los profesores responsables de cada asignatura completan con la ayuda de la coordinadora de grado, la gestora y los técnicos en simulación.

Una vez completada la "hoja de ruta" y aproximadamente un mes antes de la actividad, la coordinadora de grado se reúne con el profesor responsable de la asignatura y todos los profesores que vayan a participar en la simulación para ofrecerles una formación más dirigida y concreta. Además, a lo largo del año, se organizan pequeños cursos teórico-prácticos de formación para profesores en los que se explican aspectos básicos de la simulación, haciendo hincapié en el desarrollo de un buen briefing y debriefing.

La práctica diseñada, sea un taller de habilidades o un escenario clínico, se ensaya previamente para detectar posibles fallos y corregirlos antes de su implementación con los alumnos. Un escenario permite entrenar múltiples situaciones, cualquiera que sea el objetivo pedagógico, requiriendo para su organización y diseño un trabajo metódico y el empleo de numerosos recursos. Además, la coordinadora asiste el primer día de todas las actividades para asegurarse de que se desarrolla de acuerdo al plan previsto y se consiguen los objetivos docentes deseados. En caso de que fuera necesaria alguna mejora, las sugerencias se presentan al profesor responsable de la asignatura y se reanuda el trabajo en equipo entre los profesores y los profesionales del CSM.

Las herramientas docentes en simulación cambian a un ritmo vertiginoso, marcado por los avances en evidencias metodológicas, aplicaciones online, así como cambios tecnológicos. Ello requiere una actualización permanente del equipo docente, al menos de aquellos que desean incorporar nuevos avances y mejoras tecnológicas en sus clases. Con este objetivo, la Unidad de Educación Médica de la Facultad de Medicina implementó el CINEXEM (Curso de Innovación y Experiencia en Educación Médica:

https://www.unav.edu/web/unidad-de-educacion-medica/cinexem, que ha demostrado fomentar la creatividad y capacidad de innovación de los profesores.

En cuanto a la medición de resultados, en nuestro Centro de Simulación la evaluación es formativa. Nuestro objetivo es que aprendan el procedimiento u objetivo de aprendizaje marcado. No salen de la práctica sin haber completado el aprendizaje y sin que el profesor lo supervise, y dé un feedback de aprendizaje constructivo; pero como cada alumno es diferente y lo es su curva de aprendizaje, si necesita más tiempo pueden seguir practicando. El alumno es dueño de su aprendizaje, por ello, además de realizar los talleres de habilidades con el profesor de cada una de 
las asignaturas, a lo largo del curso tiene la posibilidad de acudir a sesiones específicas para poder practicar cuantas veces quiera. A estas actividades se les llama "Práctica libre" y "Recuerdas cómo". Pueden reservar salas y simuladores en el Centro de Simulación para practicar bien solos o en equipo (Práctica libre). También, si es necesario, pueden pedirnos que haya un docente supervisando la actividad en cuyo caso la denominamos "Recuerdas cómo". Entendemos que esta es la forma de que todos los alumnos alcancen la capacitación, individualizando la curva de aprendizaje.

\section{El paciente estandarizado como herramienta para la docencia en simulación}

Un paciente estandarizado (PE) es una persona entrenada para representar el papel de paciente en un escenario simulado y repetir con fidelidad la sintomatología y la actitud que el instructor le haya indicado. En los escenarios con simuladores, también llamamos PE a la persona que representa el papel de familiar o acompañante. La incorporación de PE a la docencia en simulación permite a los alumnos entrenar, no solo sus competencias clínicas, sino, además, sus habilidades de comunicación. Alcanzar los objetivos docentes depende, en gran medida, de la formación que reciben los PE, que debe ser exigente y minuciosa a fin de conseguir el mayor realismo posible durante el escenario y un feedback constructivo durante el debriefing.

La Universidad de Navarra cuenta con un Programa de PE desde el curso 2015-16. A lo largo de los años, el programa se ha ido depurando y consolidando hasta alcanzar el formato actual, que cuenta con un amplio número de colaboradores. Para representar el papel de enfermería en los escenarios, se cuenta con un grupo específico de profesionales de esa área.

$\mathrm{Al}$ inicio del curso académico, el CSM hace un llamamiento para reclutar nuevos PE entre los estudiantes y trabajadores de la Universidad de Navarra. Quedan excluidos, de este llamamiento, los alumnos de las facultades de Medicina y Enfermería (salvo para representar el papel de enfermera) y cualquier persona vinculada a los alumnos que van a participar en los talleres. El objetivo de esta exclusión no es otro que dotar al escenario del mayor realismo posible y evitar conflictos de interés. En el proceso de selección de los PE la experiencia en interpretación es un importante factor a tener en cuenta. De hecho, uno de los objetivos a corto plazo es reclutar nuevos PE en escuelas de teatro del área de Pamplona.

Los profesores responsables de la práctica son los encargados de elaborar el guion de su escenario con el asesoramiento de la coordinadora de grado y el equipo del CSM. Una vez definidos los objetivos de aprendizaje, y teniendo claras las necesidades de los profesores, se eligen los PE que participarán en el escenario. Si las condiciones lo permiten, es recomendable formar a más de un PE para cada escenario, ya que ello nos ofrecerá cierto margen de actuación ante imprevistos (incompatibilidades horarias, conflicto de interés, bajas...).

La labor de formar a los PE recae en los profesores encargados de la práctica, para lo que cuentan también con la colaboración de todo el equipo del CSM.

Habitualmente, un PE participa varias veces en el mismo escenario (para diferentes grupos de alumnos). Por este motivo es fundamental que, durante la formación, los PE entiendan que tendrán que repetir su actuación de la manera más fidedigna posible tanto en la forma como en el fondo. En las reuniones de formación también se pueden abordar aspectos del debriefing a fin de que el PE ofrezca una crítica constructiva al estudiante después del escenario.

Los PE perciben una retribución económica por su colaboración, que incluye el tiempo de formación y el tiempo de los escenarios. Con frecuencia, especialmente entre los PE de mayor edad, no es esa retribución la que motiva la participación en el programa de PE, si no el deseo de colaborar en la formación de futuros médicos.

En el curso 2021-22 llevamos a cabo un estudio para comparar las sensaciones y el aprendizaje de los alumnos en un escenario simulado con un PE o un paciente real. Una de las primeras conclusiones de ese estudio fue que los alumnos no eran capaces de distinguir si un paciente era real o estandarizado, y que esa incertidumbre les conducía a comportarse como lo harían con un paciente real, lo que, en palabras de los estudiantes, implica un mayor compromiso y responsabilidad. El hecho de que los alumnos no fueran capaces de distinguir entre un paciente real y un PE nos llevó a reflexionar sobre la importancia de la formación que reciben los PE (y que no habían recibido los 
pacientes reales), hecho que se vio confirmado al constatar que los alumnos valoraban mejor el debriefing recibido de los PE.

La participación de pacientes reales en escenarios simulados tiene un gran valor, ya que permite la detección de signos clínicos en la exploración física y requieren poca o ninguna caracterización. Sin embargo, a partir de nuestros hallazgos, creemos que los pacientes reales también deben recibir formación específica sobre su actuación durante el escenario y el debriefing.

A falta de un análisis más profundo de nuestros resultados, creemos que ese trabajo sirve para apoyar la idea de que los PE son perfectamente válidos para que los alumnos del Grado de Medicina entrenen sus competencias clínicas y sus habilidades de comunicación siempre que hayan recibido una formación adecuada. Como aspecto novedoso, este trabajo abrió un debate en torno a si es o no adecuado que los alum- nos desconozcan si el paciente de su escenario es real o estandarizado. La falta de transparencia con los alumnos rompería el clima de seguridad y confianza tan necesario en la simulación. Sin embargo, podría argumentarse que la línea que separa un paciente real de un PE es muy fina; ¿acaso no somos todos "pacientes" en algún momento? Teniendo esto presente, optamos por que, antes de iniciar una actividad, se informe a los alumnos de que "los pacientes que participan en los escenarios simulan una situación clínica, padezcan o no la enfermedad en cuestión".

Recientemente, se ha editado un "Manual de casos con Pacientes Estandarizados para el EC

\section{Asignatura propia}

En el CSM se imparte una asignatura optativa de 3 ETCS para alumnos de $5^{\circ}$ y $6^{0}$ cuya misión es enseñar en qué consiste y cómo se trabaja en un centro de simulación desde dentro. El objetivo es que se motiven con la docencia y con la simulación trabajando desde la mente y la visión de los instructores y técnicos. Con ello hemos conseguido que cada año tengamos más solicitudes para ser alumnos internos en el último año de grado, con la posibilidad de desarrollar su trabajo de fin de grado y de colaborar en la docencia de los alumnos de primeros cursos. Esto se ha visto reflejado en comunicaciones a congresos y publicaciones.

\section{Colaboración CSM-Laboratorio de Ingeniería Biomédica}

La Facultad de Medicina cuenta con un Laboratorio de Ingeniería Biomédica. Con ellos el CSM trabaja estrecha- mente. Esta colaboración se traduce en: elaboración de material para los distintos talleres, diseño de prototipos, propuesta de ideas y colaboración en investigación...

Semanalmente se mantiene una sesión conjunta donde se analiza la evolución de los proyectos en marcha y se van planteando nuevas propuestas y las necesidades y posibilidades de desarrollarlas.

Es muy importante, ante cualquier proyecto, contar con el asesoramiento y supervisión constante de aquellos profesionales que van a emplear en la docencia los nuevos materiales. Este aspecto es básico para que el trabajo y esfuerzo sea eficaz, útil y de calidad.

\section{El CSM y el ECOE}

El CSM ocupa un lugar muy importante en el Examen de Competencias Objetivo Estructurado (ECOE) que la Universidad de Navarra lleva a cabo en $5^{\circ}$ y $6^{\circ}$ del Grado de Medicina. Concretamente, 12 de las 21 estaciones que componen en el ECOE son estaciones para evaluar la adquisición de habilidades y se desarrollan en el propio CSM. Seis estaciones más son pruebas con PE y se llevan a cabo fuera del CSM, pero dentro de la Facultad de Medicina, gracias a una estructura de paneles desmontables con los que se reproducen salas de consulta.

En cada una de las pruebas de habilidades que se desarrollan en el CSM hay un profesor encargado de dar las instrucciones al alumno y cumplimentar la rúbrica de evaluación. Es de los pocos momentos en que se lleva a cabo una evaluación sumativa. En el caso de las pruebas con PE, las instrucciones se colocan fuera de la consulta y es el propio PE quien rellena la rúbrica. La evaluación del alumno en el ECOE resulta de la suma de su puntuación en cada una de las estaciones y, por lo tanto, podría verse afectada por la variabilidad inter-evaluador. A fin de conseguir la 
máxima reproducibilidad posible, tanto profesores como PE reciben formación específica en la evaluación del alumno y para cumplimentar las rúbricas.

Agradecimientos: A la Facultad de Medicina y Ciencias de la Salud de la Universidad de Alcalá de Henares por invitarnos a colaborar en este proyecto. A la Facultad de Medicina de la Universidad de Navarra por apoyarnos constantemente. A nuestras alumnas y alumnos, que son el centro de nuestra labor docente y dan sentido a nuestro trabajo. A todos aquellos que hacen que la simulación sea una herramienta docente fundamental en nuestras Facultades.

Contribución de los autores: J Pueyo y N Uruñuela han coordinado y diseñado este artículo, y han escrito acerca del funcionamiento general; y junto a N Díez y C Honorato han desarrollado la parte de metodología docente, quien, a su vez con D Valencia, R Orio y JC Díez han elaborado la dedicada a los técnicos. N Martín elaboró pacientes estandarizados y ECOE. S Fernández y J Alcalde realizaron la corrección definitiva.

Conflictos de Intereses: Los autores no declaran conflicto de intereses.

(C) 2021 por los autores; Esta obra está sujeta a la licencia de Reconocimiento 4.0

Internacional de Creative Commons. Para ver una copia de esta licencia, visite http://creativecommons.org/licenses/by-nc-nd/4.0/. 